PROCEEDINGS OF THE

AMERICAN MATHEMATICAL SOCIETY

Volume 128, Number 10, Pages 3025-3029

S 0002-9939(00)05347-8

Article electronically published on April 28, 2000

\title{
SMALL FORCING CREATES NEITHER STRONG NOR WOODIN CARDINALS
}

\author{
JOEL DAVID HAMKINS AND W. HUGH WOODIN
}

(Communicated by Carl G. Jockusch, Jr.)

\begin{abstract}
After small forcing, almost every strongness embedding is the lift of a strongness embedding in the ground model. Consequently, small forcing creates neither strong nor Woodin cardinals.
\end{abstract}

The widely known Levy-Solovay Theorem LevSol67 asserts that small forcing does not affect the measurability of any cardinal. (Specifically, if a forcing notion $\mathbb{P}$ has size less than $\kappa$, then $\kappa$ is measurable in the forcing extension $V^{\mathbb{P}}$ if and only if it is measurable in the ground model $V$, all the measures on $\kappa$ in $V$ extend uniquely to measures on $\kappa$ in $V^{\mathbb{P}}$, all the corresponding ultrapower embeddings lift uniquely from $V$ to $V^{\mathbb{P}}$, and all the measures on $\kappa$ in $V^{\mathbb{P}}$ arise in this way.) The argument generalizes to the other large cardinals whose existence is witnessed by the existence of certain kinds of ultrapowers, such as strongly compact cardinals, supercompact cardinals, huge cardinals, and so on.

Historically, the idea that large cardinals are invariant under small forcing addressed Gödel's hope that their existence would settle the Continuum Hypothesis. Since one can quite easily force the Continuum Hypothesis to hold or to fail via small forcing, the conclusion is inescapable that the existence of large cardinals simply has no bearing on the Continuum Hypothesis. In this sense, LevSol67 is a landmark theorem marking a change in expectations of what large cardinals would achieve.

Missing, however, from the scope of the generalizations of the theorem have been both the strong and Woodin cardinals, whose embeddings are not simple ultrapowers, but extender embeddings, directed systems of ultrapowers. It was widely expected among set theorists that the situation for these cardinals would accord with [LevSol67, though initially no proof was forthcoming. Several years ago, however, the second author of this paper was able successfully to treat these extender embeddings and prove that small forcing creates neither strong nor Woodin cardinals. Since the proof differs fundamentally from [LevSol67] and furthermore seems not to be widely known, we would like to present it here in a precise level-by-level version which obtains the result also for cardinals which are only partially strong.

Received by the editors September 1, 1998 and, in revised form, November 9, 1998.

1991 Mathematics Subject Classification. Primary 03E55, 03E40.

Key words and phrases. Small forcing, large cardinals, strong cardinal, Woodin cardinal.

The research of the first author has been supported in part by grants from the PSC-CUNY Research Foundation, and of the second author, by grants from the NSF. 
Main Theorem. After small forcing, a cardinal $\kappa$ is strong if and only if it was strong in the ground model.

Level-by-level Version. If $g \subseteq \mathbb{P}$ is $V$-generic for the small forcing $\mathbb{P}$, then for every ordinal $\lambda$ (except possibly the limit ordinals with $\operatorname{cof}(\lambda) \leq|\mathbb{P}|^{+}$), every natural $\lambda$-strongness embedding $j: V[g] \rightarrow M[g]$ in the extension lifts a $\lambda$-strongness embedding $j \uparrow V: V \rightarrow M$ definable in the ground model.

Corollary. After small forcing, a cardinal $\theta$ is Woodin if and only if it was Woodin in the ground model.

Let us define the relevant concepts. A forcing notion $\mathbb{P}$ is small relative to $\kappa$ when $|\mathbb{P}|<\kappa$. A cardinal $\kappa$ is strong when for every $\lambda$ it is $\lambda$-strong, i.e. there is a $\lambda$-strongness embedding $j: V \rightarrow M$, one with critical point $\kappa$ and $V_{\lambda} \subseteq M$. A cardinal $\theta$ is Woodin when for every $A \subseteq \theta$ there is a $\kappa<\theta$ which is $<\theta$-strong for $A$, meaning that for every $\lambda<\theta$ there is an embedding $j: V \rightarrow M$ with critical point $\kappa$ such that $j(A) \cap \lambda=A \cap \lambda$; such embeddings are said to be $\lambda$ strong for $A$. By coding extra information into the set $A$, one can ensure that there is such a $j$ which is $\lambda$-strong. A $\lambda$-strongness embedding $j: V \rightarrow M$ is natural when $M=\left\{j(h)(s) \mid h \in V \& s \in \gamma^{<\omega}\right\}$, where $\gamma=\left|V_{\lambda}\right|^{M}$. Equivalently, we could require that $M=\left\{j(h)(s) \mid h \in V \& s \in V_{\lambda}\right\}$.

Before taking up the theorem, let us prove a few general facts about strongness embeddings. First, we claim that every strongness embedding factors through a natural embedding. To see this for any particular $\lambda$-strongness embedding $j$ : $V \rightarrow M$, where $\lambda>\kappa$, let $X=\left\{j(h)(s) \mid h \in V \& s \in \gamma^{<\omega}\right\}$. By checking the Tarski-Vaught criterion, it is easy to see that $X$ is an elementary substructure of $M$ covering $\operatorname{ran}(j)$, and so if $\pi: X \rightarrow M_{0}$ is the Mostowski collapse of $X$, we obtain a map $j_{0}: V \rightarrow M_{0}$ by simply defining $j_{0}=\pi \circ j$. Thus also $j=k \circ j_{0}$, where $k=\pi^{-1}$, and so $j$ factors through $j_{0}$.

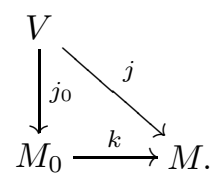

Since $V_{\lambda} \subseteq X$, it follows that $\pi " V_{\lambda}=V_{\lambda}$ and $V_{\lambda} \subseteq M_{0}$, and since $M_{0}=\operatorname{ran}(\pi)$ and $\pi\left\lceil\gamma=i d\right.$, it follows that $M_{0}=\left\{j_{0}(h)(s) \mid h \in V \& s \in \gamma^{<\omega}\right\}$. Thus, $j_{0}$ is a natural $\lambda$-strongness embedding, as desired. Note that if $j$ is $\lambda$-strong for $A$, then so also is $j_{0}$, because the critical point of $k$ is at least $\lambda$. The point is therefore that when $\theta$ is a Woodin cardinal, one can take the witnessing embeddings to be natural.

Second, while strongness embeddings in general need not satisfy any closure properties, the natural strongness embeddings are much better behaved. Specifically, we claim that if $j: V \rightarrow M$ is a natural $\lambda$-strongness embedding with critical point $\kappa$ and $\lambda$ is either a successor ordinal or a limit ordinal of cofinality above $\kappa$, then $M$ is closed under $\kappa$-sequences. Otherwise, $M$ is closed under $<\operatorname{cof}(\lambda)$-sequences. To see this, we may suppose that $\lambda>\kappa$ and $M$ is equal to $\left\{j(h)(s) \mid h \in V \& s \in V_{\lambda}\right\}$. In the first case, suppose that $\lambda=\xi+1$ and $\left\langle j\left(h_{\alpha}\right)\left(s_{\alpha}\right) \mid \alpha<\kappa\right\rangle$ is a $\kappa$-sequence of elements from $M$, with each $s_{\alpha} \in V_{\xi+1}$. Since a $\kappa$-sequence of subsets of $V_{\xi}$ can be coded with a single subset of $V_{\xi}$, it follows that $\left\langle s_{\alpha} \mid \alpha<\kappa\right\rangle$ is in $M$. Then, since the sequence $\left\langle j\left(h_{\alpha}\right) \mid \alpha<\kappa\right\rangle=j\left(\left\langle h_{\alpha} \mid \alpha<\kappa\right\rangle\right) \uparrow \kappa$ is in $M$, it follows that 
$\left\langle j\left(h_{\alpha}\right)\left(s_{\alpha}\right) \mid \alpha<\kappa\right\rangle$ is in $M$, as desired. For the next case, when $\lambda$ is a limit ordinal of cofinality larger than $\kappa$, then on cofinality grounds the sequence $\left\langle s_{\alpha} \mid \alpha<\kappa\right\rangle$ is in $V_{\lambda}$, and hence in $M$, so again $\left\langle j\left(h_{\alpha}\right)\left(s_{\alpha}\right) \mid \alpha<\kappa\right\rangle$ is in $M$, as desired. Finally, if $\lambda$ is a limit ordinal, $\beta<\operatorname{cof}(\lambda) \leq \kappa$ and $\left\langle j\left(h_{\alpha}\right)\left(s_{\alpha}\right) \mid \alpha<\beta\right\rangle$ is a sequence of elements from $M$, then again on cofinality grounds we know $\left\langle s_{\alpha} \mid \alpha<\beta\right\rangle$ is in $V_{\lambda}$, and hence in $M$, and so $\left\langle j\left(h_{\alpha}\right)\left(s_{\alpha}\right) \mid \alpha<\beta\right\rangle$ is in $M$, as desired.

Now we are ready to prove the main theorem.

Main Theorem. Suppose $g \subseteq \mathbb{P}$ is $V$-generic for forcing $\mathbb{P}$ of size less than $\kappa$. Then $\kappa$ is strong in $V$ if and only if it is strong in $V[g]$.

Proof. We may assume that $\mathbb{P} \in V_{\kappa}$. Let $\delta=|\mathbb{P}|<\kappa$. The forward direction of the theorem is trivial because any $\lambda$-strongness embedding $j: V \rightarrow M$ in $V$ lifts to an embedding $j: V[g] \rightarrow M[g]$ in $V[g]$ by simply defining $j\left(\tau_{g}\right)=j(\tau)_{g}$ for any name $\tau \in V$. This embedding witnesses the $\lambda$-strongness of $\kappa$ in $V[g]$ because $V[g]_{\lambda}=V_{\lambda}[g]$. For the converse, suppose that $\kappa$ is $\lambda$-strong in $V[g]$ with natural embedding $j: V[g] \rightarrow M[g]$, where $\lambda>\kappa$ is either a successor ordinal or a limit ordinal with $\operatorname{cof}(\lambda)>\delta^{+}$. To prove the theorem, we will show that $j\lceil V: V \rightarrow M$ is definable in $V$ and witnesses there that $\kappa$ is $\lambda$-strong. Since $j$ is natural, we know that $M[g]=\left\{j(h)(s) \mid h \in V[g] \& s \in \gamma^{<\omega}\right\}$, where $\gamma=\beth_{\lambda}^{M}$. It is easy to verify that $V_{\kappa}=\left(V_{\kappa}\right)^{M}$ because sets with rank below the critical point are fixed by $j$. Moreover, if $A \subseteq \kappa$ in $V$, then $A=j(A) \cap \kappa$ and so $P(\kappa)^{V} \subseteq M$. Conversely if $A \subseteq \kappa$ in $M$, then every initial segment of $A$ is in $V$ and so $A$ must also be in $V$ since the forcing is $\kappa$-c.c. It follows that $V_{\kappa+1}=\left(V_{\kappa+1}\right)^{M}$. Let $E$ be the induced $V$-extender, namely, $E=\left\{\langle A, s\rangle \mid s \in \gamma^{<\omega} \& A \in V_{\kappa+1} \& s \in j(A)\right\}$.

We claim that the restricted embedding $j \uparrow V: V \rightarrow M$ is precisely the embedding induced by the extender $E$. It suffices to show that $M$ is equal to $\left\{j(h)(s) \mid h \in V \& s \in \gamma^{<\omega}\right\}$, for then the map which takes the $E$-equivalence class of $\langle h, s\rangle$ in $\operatorname{Ult}(V, E)$ to $j(h)(s)$ will be an isomorphism. By the assumption on $j$ we know that any set $a$ in $M$ has the form $j(h)(s)$ for some function $h: \kappa^{n} \rightarrow V$ in $V[g]$ and some $s \in \gamma^{n}$ and $n \in \omega$; the difficulty is to find such a function $h$ in $V$. But since $h=\dot{h}_{g}$ for some name $\dot{h} \in V$ and $j(\dot{h})_{g}(s)=a$, there must be a condition $p \in g$ which forces over $M$ that $j(\dot{h})(\check{s})=\check{a}$. In $V$ define the function $\bar{h}(x)=y$ when $p$ forces that $\dot{h}(\check{x})=\check{y}$. It follows that $j(\bar{h})(s)=a$, and so $a$ has the desired form.

The point now is that in order to show that $j \uparrow V$ is definable (from parameters) in $V$, it suffices to show that $E$ is in $V$. For this, we will first prove the claim that if $F \subseteq E$ and $|F|=\delta$, then there is $F^{*} \in V$ such that $F \subseteq F^{*} \subseteq E$. Fix such an $F$, and let $\sigma$ be the set of generators which appear in $F$, that is, the set of ordinals appearing in any $s$ which appears in $F$. So $\sigma \subseteq \gamma$ and $|\sigma| \leq \delta$. Consequently, by the hypothesis on $\lambda$ and the remarks on the closure of natural strongness embeddings, $\sigma$ is in $M[g]$. So the set $\sigma$ has names in both $M$ and $V$. By iteratively using such names from $V$ and $M$, we may construct an increasing $\delta^{+}$sequence of sets $\vec{\sigma}=\left\langle\sigma_{\alpha} \mid \alpha<\delta^{+}\right\rangle$ beginning with $\sigma_{0}=\sigma$, such that every $\sigma_{\alpha}$ has cardinality $\delta$, for cofinally many $\alpha$ the set $\sigma_{\alpha}$ is in $V$, and for cofinally many $\alpha$ it is in $M$. Let $\tau=\bigcup_{\alpha} \sigma_{\alpha}$. Since $\vec{\sigma} \in M[g]$ by the closure of the embedding, the sequence has names in both $M$ and $V$. In $V$ there are conditions in $\mathbb{P}$ which force the various $\sigma_{\alpha}$ to appear, and so since $\delta^{+}$is regular there must be a single condition which decides unboundedly many of the $\dot{\sigma}_{\alpha}$ in $\dot{\vec{\sigma}}$. Thus, $\tau \in V$. Similarly, using a name in $M$ for $\vec{\sigma}$ there must be a single condition deciding unboundedly many of the $\sigma_{\alpha}$, and so $\tau \in M$. Let 
$\mu=\left\{A \in V_{\kappa+1} \mid \tau \in j(A)\right\}$. Since this is a $V$-measure in $V[g]$, the Levy-Solovay Theorem for measurable cardinals tells us that it lies in $V$. From $\mu$ and $\tau$ we can in $V$ compute the set $F^{*}=\left\{\langle A, s\rangle \mid s \in \tau^{<\omega} \& A \in V_{\kappa+1} \& s \in j(A)\right\}=E\lceil\tau$, since any such $s$ corresponds to a certain projection of $\tau$. That is, if $s$ consists of the $\alpha_{0}^{\text {th }}, \ldots, \alpha_{n}^{\text {th }}$ elements of $\tau$, then $\langle A, s\rangle \in F^{*}$ exactly when $\pi^{-1} A \in \mu$ where $\pi(t)$ restricts $t$ to its $\alpha_{0}^{\text {th }}, \ldots, \alpha_{n}^{\text {th }}$ elements. Consequently, $F^{*}$ is in $V$, as we claimed.

Now let us show that $E \in V$. Since $E \in V[g]$, it has a name $\dot{E}$ in $V$. Suppose $\eta \gg \gamma$ and let $X \prec V_{\eta}$ be an elementary substructure of size $\delta$ which contains $\mathbb{P}$, $F^{*}, \dot{E}$ and every element of $\mathbb{P}$. It follows that $g$ is $X$-generic, that $X[g] \prec V_{\lambda}[g]$ and that $E \in X[g]$. Let $F=E \cap X[g]$. By the previous paragraph, there is a set $F^{*} \in V$ such that $F \subseteq F^{*} \subseteq E$. Since $F=F \cap X \subseteq F^{*} \cap X \subseteq E \cap X=F$, it follows that $F=F^{*} \cap X$ in $V$. Thus, there must be a condition $p \in g$ which decides " $\langle A, s\rangle \in \dot{E}$ " for all $\langle A, s\rangle$ in $X$. By elementarity, therefore, $p$ decides " $\langle A, s\rangle \in \dot{E}$ " for all $\langle A, s\rangle$ in $V$. So $E$ lies in $V$.

We have argued that the restricted embedding $j: V \rightarrow M$ is therefore definable from $E$ in $V$. In order to finish the proof of the theorem, then, it suffices for us to show that $V_{\lambda} \subseteq M$. Certainly $V_{\lambda} \subseteq M[g]$ since the full embedding was $\lambda$-strong. Thus, for any $a \in V_{\lambda}$ there is a name $\dot{a} \in M$ and a condition $p \in g$ which forces (over $V$ ) that $\check{a}=\dot{a}$. It follows using $p$ and $\dot{a}$ in $M$ that $a \in M$. That is, $V_{\lambda} \subseteq M$. Thus, the restricted embedding $j: V \rightarrow M$ witnesses that $\kappa$ is $\lambda$-strong in $V$.

The proof actually established the level-by-level version that we stated in the beginning of this paper:

Level-by-level Version. If $g \subseteq \mathbb{P}$ is $V$-generic for the small forcing $\mathbb{P}$, then for every ordinal $\lambda$ (except possibly the limit ordinals with $\operatorname{cof}(\lambda) \leq|\mathbb{P}|^{+}$), every natural $\lambda$-strongness embedding $j: V[g] \rightarrow M[g]$ in the extension lifts a $\lambda$-strongness embedding $j \uparrow V: V \rightarrow M$ definable in the ground model.

We are left with the intriguing possibility that small forcing could make $\mathrm{a}<\lambda$ strong cardinal $\lambda$-strong when $\lambda$ is a limit ordinal with small cofinality. Certainly it seems possible that if there are several kinds of $\lambda$-extenders in the ground model, then after adding a Cohen real, say, one could consult the new real $x$ and glue together various pieces of these extenders in such a way that the resulting embedding $j: V[x] \rightarrow M[x]$ could not be the lift of an embedding from the ground model; the idea being that from the restricted embedding $j: V \rightarrow M$ one would be able to recover the real $x$. In such a situation, the strict analogue of the Levy-Solovay theorem for strongness embeddings would fail, because not every natural strongness embedding would lift an embedding from the ground model. More difficult, however, is the question of whether after small forcing one could glue together various $<\lambda$-strongness extenders to make a $\lambda$-strong embedding in the extension even when no $\lambda$-strong embedding exists in the ground model. That is, the following question remains open:

Question. Can small forcing increase the degree of strongness of a cardinal?

Of course, the only possibility for an affirmative answer is that a $<\lambda$-strong cardinal $\kappa$ is made $\lambda$-strong in the extension for some limit ordinal of small cofinality.

Let us now treat the case of Woodin cardinals.

Corollary. After small forcing, a cardinal is Woodin if and only if it was Woodin in the ground model. 
Proof. As in the theorem, a trivial lifting argument shows that small forcing cannot destroy any Woodin cardinal. Conversely, suppose that $\theta$ is Woodin in $V[g]$, a small forcing extension, and that $A \subseteq \theta$ in $V$. There must be a cardinal $\kappa<\theta$ such that for every $\lambda<\theta$ there is a natural $\lambda$-strongness embedding $j: V[g] \rightarrow M[g]$ with critical point $\kappa$ such that $j(A) \cap \lambda=A \cap \lambda$. If $\lambda$ is a successor ordinal, then by the Level-by-Level version of the Main Theorem the restricted embedding $j: V \rightarrow M$ satisfies the same hypothesis in $V$. Thus, since the successor ordinals are unbounded in $\theta$, this means that $\theta$ is Woodin in $V$, as desired.

\section{REFERENCES}

[LevSol67] Azriel Levy, Robert M. Solovay, Measurable cardinals and the Continuum Hypothesis, IJM 5 (1967), 234-248. MR 37:57

Mathematics Program, The Graduate Center of the City University of New York, 365 Fifth Avenue, New York, New York 10016

E-mail address: jhamkins@gc.cuny.edu

$U R L:$ http://gc. cuny.edu/Faculty/Hamkins

Department of Mathematics, University of California, Berkeley, California 94720

E-mail address: woodin@math.berkeley.edu 